\title{
Water Content and Scatterers Dispersion Evaluation in Colorectal Tissues
}

\author{
Isa Carneiro ${ }^{1}$, Sónia Carvalho ${ }^{1}$, Rui Henrique ${ }^{1,2}$, Luís Oliveira ${ }^{3,4^{*}}$, and Valery V. Tuchin ${ }^{5,6,7}$ \\ ${ }^{1}$ Portuguese Oncology Institute of Porto, Department of Pathology and Cancer Biology and Epigenetics \\ Group-Research Centre, Rua Dr. António Bernardino de Almeida S/N, 4200-072 Porto, Portugal \\ ${ }^{2}$ Department of Pathology and Molecular Immunology, Institute of Biomedical Sciences Abel Salazar - University of \\ Porto (ICBAS-UP), Rua de Jorge Viterbo Ferreira n-228, 4050-313 Porto, Portugal \\ ${ }^{3}$ Polytechnic of Porto, School of Engineering, Physics Department, Rua Dr. António Bernardino de Almeida, 431, \\ 4200-072 Porto, Portugal \\ ${ }^{4}$ Centre of Innovation in Engineering and Industrial Technology, ISEP, Rua Dr. António Bernardino de Almeida, 431, \\ 4200-072 Porto, Portugal \\ ${ }^{5}$ Research-Educational Institute of Optics and Biophotonics, Saratov State University, 83 Astrakhanskaya str., \\ Saratov 410012, Russia \\ ${ }^{6}$ Laboratory of Laser Diagnostics of Technical and Living Systems, Institute of Precision Mechanics and Control \\ Institute of the Russian Academy of Sciences, 24 Rabochaya, Saratov 410028, Russia \\ ${ }^{7}$ Department of Laser and Biotechnical Systems, Samara National Research University, 34 Moskovskoye Shosse, \\ Samara 443086, Russia
}

* e-mail: Imo@isep.ipp.pt

\begin{abstract}
Optical clearing treatments reduce light scattering in biological tissues. Due to the heterogeneous composition of tissues, such high scattering is created by the refractive index mismatch between tissue fluids and scatterers. With the objective of collecting experimental data to predict and characterize the magnitude of the refractive index matching in optical clearing treatments, a series of studies were performed with colorectal tissues. The free water content in normal mucosa, muscle and pathological colorectal tissues was estimated, showing similar values for normal tissues and about $5 \%$ more in pathological tissue. The refractive index of the tissues was measured at various wavelengths between UV and NIR. This data was used to estimate the refractive dispersion curves for the various tissues, which were then combined with the free water contents to calculate the dispersion of scatterers in these tissues. These dispersions remain unchanged during optical clearing and by obtaining such spectral data, it is possible to quantify and characterize the refractive index matching mechanism in any optical clearing treatment to be applied in these tissues. The differences obtained between the experimental data of healthy and pathological tissues can also be used as a marker for cancer progression in ex vivo analysis. (C) 2017 Journal of Biomedical Photonics \& Engineering.
\end{abstract}

Keywords: colorectal tissue; cancer; refractive index; light scattering; tissue water content; free and bound water; optical clearing.

Paper \#3254 received 30 Oct 2017; revised manuscript received 11 Dec 2017; accepted for publication 19 Dec 2017; published online 31 Dec 2017. doi: 10.18287/JBPE17.03.040301. [Saratov Fall Meeting 2017 Special Issue].

\section{References}

1. T. Vo-Dinh (ed.), Biomedical Photonics Handbook, 2nd ed., CRC Press, Boca Raton (2014).

2. I. J. Bigio, and S. Fantini, "Quantitative Biomedical Optics: Theory, Methods, and Applications," Cambridge Texts in Biomedical Engineering, Cambridge University Press, Cambridge (2016). 
3. A. Sabouni, S. Noghanian, and S. Pistorius, "Effects of tissue composition on the accuracy of microwave breast tumour imaging," IFMBE Proceedings 14, 1489-1493 (2007).

4. P. C. Miranda, L. Correia, R. Salvador, and P. J. Basser, "Tissue heterogeneity as a mechanism for localized neural stimulation by applied electric fields," Physics in Medicine and Biology 52(18), 5603-5617 (2007).

5. V. V. Tuchin, Optical Clearing of Tissues and Blood, SPIE Press, Bellingham, Washington (2006). ISBN: 9780819481108

6. L. Oliveira, A. Lage, M. P. Clemente, and V. Tuchin, "Optical characterization and composition of abdominal wall muscle from rat," Optics and Lasers in Engineering 47(6), 667-672 (2009).

7. L. Oliveira, A. Lage, M. P. Clemente, and V. V. Tuchin, "Rat muscle opacity decrease due to the osmosis of a simple mixture," Journal of Biomedical Optics 15(5), 055004 (2010).

8. V. Gilard, R. Martino, M. Malet-Martino, M. Riviere, A. Gournay, and R. Navarro, "Measurement of total water and bound water contents in human stratum corneum by in vitro proton nuclear magnetic resonance spectroscopy,” International Journal of Cosmetic Science 20(2), 117-125 (1998).

9. C. Li, J. Jiang, and K. Xu, "The variations of water in human tissue under certain compression: studied with diffuse reflectance spectroscopy," Journal of Innovative Optical Health Sciences 6(1), 1350005 (2013).

10. D. Zhu, K. V. Larin, Q. Luo, and V. V. Tuchin, "Recent progress in tissue optical clearing," Laser and Photonic Reviews 7(5), 732-757 (2013).

11. S. Carvalho, N. Gueiral, E. Nogueira, R. Henrique, L. Oliveira, and V. V. Tuchin, "Glucose diffusion in colorectal mucosa - a comparative study between normal and cancer tissues," Journal of Biomedical Optics 22(9), 091506 (2017).

12. D. K. Tuchina, R. Shi, A. N. Bashkatov, E.A. Genina, D. Zhu, Q. Luo, and V. V. Tuchin, "Ex vivo optical measurements of glucose diffusion kinetics in native and diabetic mouse skin," Journal of Biophotonics 8(4), 332-346 (2015).

13. E. A. Genina, A. N. Bashkatov, Y. P. Sinichkin, I. Yu. Yanina, and V. V. Tuchin, "Optical clearing of biological tissues: prospects of application in medical diagnosis and phototherapy," Journal of Biomedical Photonics \& Engineering 1(1), 22-58 (2016).

14. E. A. Genina, A. N. Bashkatov, and V. V Tuchin, "Tissue optical immersion clearing," Expert Review of Medical Devices 7(6), 825-842 (2010).

15. L. Oliveira, M. I. Carvalho, E. Nogueira, and V. V. Tuchin, "Optical clearing mechanisms characterization in muscle," Journal of Innovative Optical Health Sciences 9(5), 1650035 (2016).

16. L. Oliveira, M. I. Carvalho, E. M. Nogueira, and V. V. Tuchin, "The characteristic time of glucose diffusion measured for muscle tissue at optical clearing," Laser Physics 23(7), 075606 (2013).

17. D. Tuchina, A. Bashkatov, A. Bucharskaya, E. Genina, and V. Tuchin, "Study of glycerol diffusion in skin and myocardium ex vivo under the conditions of developing alloxan-induced diabetes," Journal of Biomedical Photonics \& Engineering 3(2), 020302 (2017).

18. A. Sdobnov, M. E. Darvin, J. Lademann, and V. Tuchin, "A comparative study of ex vivo skin optical clearing using two-photon microscopy," Journal of Biophotonics 10(9), 1115-1123 (2017).

19. L. Oliveira, M. I. Carvalho, E. M. Nogueira, and V. V. Tuchin, "Skeletal muscle dispersion (400 - $1000 \mathrm{~nm})$ and kinetics at optical clearing," Journal of Biophotonics, e201700094 (2017).

20. D. W. Leonard, and K. M. Meek, "Refractive indices of the collagen fibrils and extrafibrillar material of the corneal stroma," Biophysical Journal 72(3), 1382-1387 (1997).

21. S. H. Chung, A. E. Cerussi, C. Klifa, H. M. Baek, O. Birgul, G Gulsen, S. I. Merritt, D. Hsiang, and B. J. Tromberg, "In vivo water state measurements in breast cancer using broadband diffuse optical spectroscopy," Physics in Medicine and Biology 53(23), 6713-6727 (2008).

22. H. Brenner, M. Kloor, and C. P. Poxl, “Colorectal cancer," Lancet 383(9927), 1490-1502 (2014).

23. S. Carvalho, N. Gueiral, E. Nogueira, R. Henrique, L. Oliveira, and V. Tuchin, "Wavelength dependence of the refractive index of human colorectal tissues: comparison between healthy mucosa and cancer," Journal of Biomedical Photonics \& Engineering 2(4), 040307 (2016).

24. P. Giannios, S. Koutsoumpos, K. G. Toutouzas, M. Matiatou, G. C. Zografos, and K. Moutzouris, "Complex refractive index of normal and malignant human colorectal tissue in the visible and near-infrared," Journal of Biophotonics 10(2), 303-310 (2017).

25. S. Carvalho, N. Gueiral, E. Nogueira, R. Henrique, L. Oliveira, and V. V. Tuchin, "Comparative study of the optical properties of colon mucosa and colon precancerous polyps between 400 and $1000 \mathrm{~nm}$," Proceedings SPIE 10063, 100631L (2017).

26. A. Y. Sdobnov, V. V Tuchin, J. Lademann, and M. E Darvin, "Confocal Raman microscopy supported by optical clearing treatment of the skin - influence on collagen hydration," Journal of Physics D: Applied Physics 50, 285401-1-9 (2017).

27. R. Chang, "Reactions in aqueous solutions" in Chemistry, 10th ed., R. Chang (ed.), McGraw-Hill, New York (2010). ISBN: 9780078916915. 
28. A. A. Raginov, and G. N. Shcherbakova, Infusion-transfusion therapy: a guide, GEOTAR Media, Moscow (2010). ISBN: 9785970415382 [in Russian].

29. H. Li, and S. Xie, "Measurement method of the refractive index of biotissue by total internal reflection," Applied Optics 35(10), 1793-1795 (1996).

30. Q. Ye, J. Wang, Z.-C. Deng, W.-Y. Zhou, C.-P. Zhang, and J.-G. Tian, "Measurement of the complex refractive index of tissue-mimicking phantoms and biotissue by extended differential total reflection method," Journal of Biomedical Optics 16(9), 097001 (2011).

31. J.-C. Lai, Y.-Y. Zhang, Z.-H. Li, H.-J. Jiang, and A.-Z. He, "Complex refractive index measurement of biological tissues by attenuated total reflection ellipsometry," Applied Optics 49(16), 3235-3238 (2010).

32. H. Ding, J. Q. Lu, K. M. Jacobs, and X.-H. Hu, "Determination of refractive indices of porcine skin tissues and intralipid at eight wavelengths between 325 and $1557 \mathrm{~nm}$," Journal of the Optical Society of America A 22(6), 1151-1157 (2005).

33. Y. L. Jin, J. Y. Chen, L. Xu, and P. N. Wang, "Refractive index measurement for biomaterial samples by total internal reflection," Physics in Medicine and Biology 51(20), N271-N379 (2006).

34. H. Ding, J. Q. Lu, W. A. Wooden, P. J. Kragel, and X.-H. Hu, "Refractive indices of human skin tissues at eight wavelengths and estimated dispersion relations between 300 and $1600 \mathrm{~nm}$," Physics in Medicine and Biology 51(6), 1479-1489 (2006).

35. Z. Deng, J. Wang, Q. Ye, T. Sun, W. Zhou, J. Mei, C. Zhang, and J. Tian, "Determination of continuous complex refractive dispersion of biotissue based on internal reflection," Journal of Biomedical Optics 21(1), 015003 (2016).

36. https://en.wikipedia.org/wiki/Glycerol

37. Density of Glycerine-Water Solutions

38. L. Oliveira, M. I. Carvalho, E. M. Nogueira, and V. V. Tuchin, "Diffusion characteristics of ethylene glycol in skeletal muscle," Journal of Biomedical Optics 20(5), 059801 (2015).

39. G. M. Hale, and M. R. Querry, "Optical constants of water in the $200 \mathrm{~nm}$ to $200 \mu \mathrm{m}$ wavelength region," Applied Optics 12(3), 555-563 (1973).

\section{Introduction and theoretical background}

The internal composition of biological tissues is in general heterogeneous and tissue's optical properties depend on such composition and internal arrangement. Typically, soft biological tissues contain cells and their organelles, proteins and extracellular fibers that are surrounded by the cell cytoplasm and interstitial fluid (ISF), respectively [1-5]. These liquids contain mostly water and a small quantity of dissolved salts, proteins and organic compounds [5-7]. Light scattering is usually very significant in tissues. In part, such high scattering is originated by the refractive index (RI) mismatch that exists between tissue fluids and the other tissue components, which are commonly designated as scatterers [5]. As an example, for $589.6 \mathrm{~nm}$, typical values for the RI of ISF are 1.35-1.37, while the RI of scatterers ranges from 1.39 to 1.47 [5]. Higher RI mismatch is obtained if we consider tissue dry matter solid tissue components without any water. Typical values for the RI of skin melanin and dry proteins in skeletal muscle have been reported as: 1.6 [5] and 1.58 [6], respectively.

There are two types of water in biological tissues free (bulk) and bound water. Bound water, which is strongly connected to the other tissue components, can only be converted into free water when a strong or longterm stimulation is applied [6-7]. Scatterers are a combination of dry components and bound water. Free water is able to move freely inside the tissue or to the outside if stimulated to do so [8-9]. Free water can be found in the ISF or inside the tissue cells, and small magnitude or short-term stimulations can initiate its movement.

Since, in a macroscopic scale, no physical boundaries are perceived between tissue components, a biological tissue is generally considered as a continuous material, with spatial variation in the RI [5].

The RI mismatch between tissue components can be reduced by the application of optical clearing (OC) treatments. Such technique has been studied in recent years [5, 10-14], and it operates through two main mechanisms: tissue dehydration and RI matching [15]. During tissue dehydration, only the free water in the tissue will flow out to allow for the optical clearing agent (OCA) to flow from the treating solution into the interstitial space of the tissue. OCAs are innocuous chemicals that have higher RI than water, better matched to the RI of tissue scatterers. Such chemicals are in general sugars, alcohols and contrasting agents [14]. Several OC studies have been reported in literature that use OCAs such as glucose [12, 16], glycerol [7, 17], dimethyl sulfoxide, mannitol, propylene glycol and $\mathrm{x}$ ray contrast agents, such as Trazograph ${ }^{\mathrm{TM}}$ and Omnipaque $^{\mathrm{TM}}[14,18]$. A significant decrease in tissue scattering properties or an increase in tissue depth and in image contrast when using imaging techniques has been demonstrated in these studies [10].

Due to the exchange between the free water in tissue fluids and the OCA in the treating solution, an increase in the RI of the ISF will occur. The RI of tissue scatterers will remain unchanged if no strong or no long-term treatment is applied. If the RI of scatterers is 
known, the calculation of the RI change in the ISF can be made to characterize the RI matching for a particular treatment [19]. After calculating the time dependence for the RI of the ISF, the time dependence for the RI of the tissue as a whole can be calculated using Gladstone and Dale equation [20]. The same equation can also be used to calculate the RI of the dry matter or scatterers in the tissue, provided that the bound and free water contents are known. It is highly important to know the total and free water content in biological tissues to quantify light scattering and absorption and to plan efficient OC treatments to reduce light attenuation. Normal and malignant tissues have been reported to have different free and bound water contents [11, 21], and though the knowledge of these values can be considered as a marker of cancer progression in general. The refractive dispersion curves of tissue scatterers and dry matter are also necessary for treatment planning and characterization or even for diagnostic purposes.

Human colorectal tissues are of great interest for research due to the great incidence of colorectal cancer in worldwide populations [22]. Several studies have been recently performed with these tissues to evaluate their dispersion curves [23-24], to estimate their optical properties [25] or to quantify the OCA diffusion properties in OC treatments [11]. On the other hand, these tissues are not fully studied, and the quantification of free and bound water contents or the estimation of the refractive dispersion curves for scatterers and dry matter were not obtained so far.

Considering these aspects, it was our objective to perform experimental studies that would lead to the determination of the free water content in colorectal tissues and to the estimation of the dispersion curves of their scatterers. The free water content in soft tissues is typically around $60 \%$ (water volume per tissue volume) $[11,15-16]$. To determine the correct water content in ex vivo tissues, one can use $\mathrm{OC}$ treatments. If a tissue is treated with a solution that contains the same amount of water as the free water in the tissue, there will be a water balance between the treating solution and the tissue and only the OCA in the solution will flow into the tissue. As we have done for other previous research studies with other tissues [11, 15-16], to identify the free water content, we have used as probe OCAs molecules of glucose, glycerol or ethylene glycol in water solutions with various concentrations between $20 \%$ to $60 \%$. Since OCAs are hyperosmotic agents [18, $26]$, instead of using the OCA concentration in aqueous solution, one can use the osmolarity concept to identify a solution by its clearing strength. The molar concentration of the OCA in a solution is calculated as the number agent moles $(n)$ per solution volume $(V)$ [27]:

$$
C_{M}=\frac{n}{V}
$$

The molar concentration described by Eq. (1) is usually represented in mol/l. On the other hand, if we know the molar mass $(M)$ of an OCA and the density of
OCA-water solutions $(\rho)$, one can re-arrange equation to 1 calculate molar concentration:

$$
C_{M}=\frac{\rho}{M}
$$

For nonelectrolytes, the molar concentration $(1 \mathrm{~mol} / \mathrm{l})$ is equal to osmolarity $(1 \mathrm{osmol} / \mathrm{l})$ [28]. In the present study, we have used only glycerol to prepare the OC solutions to treat the colorectal muscle samples. The glycerol concentrations in water solutions and the calculated osmolarities, are presented in Sub-section 2.3 .

Several and independent studies that include direct tissue RI measurements for some wavelengths were performed. The methods used in these studies are described in Section 2.

\section{Materials and methods}

The research presented in this study was performed using different methods. In the studies to obtain the refractive dispersions of tissues, normal and pathological colorectal tissues were used. In the OC studies to determine the free water content, only colorectal muscle tissues were used. The normal colorectal mucosa and the pathological tissues have been previously studied and we used these already published data [11] to calculate the free water content.

Sub-section 2.1 presents a description of the different tissues samples used in this research and the methods used to prepare them. Sub-section 2.2 describes the method to obtain the refractive dispersion curves for all tissues studied and Sub-section 2.3 presents a description of the method used to estimate the free water content in tissues. The calculation procedure to obtain the refractive dispersions for tissue scatterers is described in Sub-section 2.4.

\subsection{Tissue samples}

Human healthy and pathological colorectal samples used in this study were collected from 7 adult patients undergoing colorectal surgery at Portuguese Oncology Institute of Porto, Portugal. The patients have signed a written consent previous to the surgical procedures, allowing for subsequent use of surgical specimens for diagnostic and research purposes. Such agreement has been approved by the Ethics Committee of the Portuguese Oncology Institute of Porto, Portugal.

Fig. 1 represents a human colorectal histology section and the gross features of an invasive colorectal cancer.

After collecting the samples from surgical procedures, the pathological and healthy areas were separated and preserved frozen at $-80{ }^{\circ} \mathrm{C}$ for a period of 12 to $24 \mathrm{hrs}$. After this period, the samples were prepared for each type of study as described below and immediately used. Prior to each set of measurements, the samples were kept in saline for $10 \mathrm{~min}$ to mimic natural hydration. 


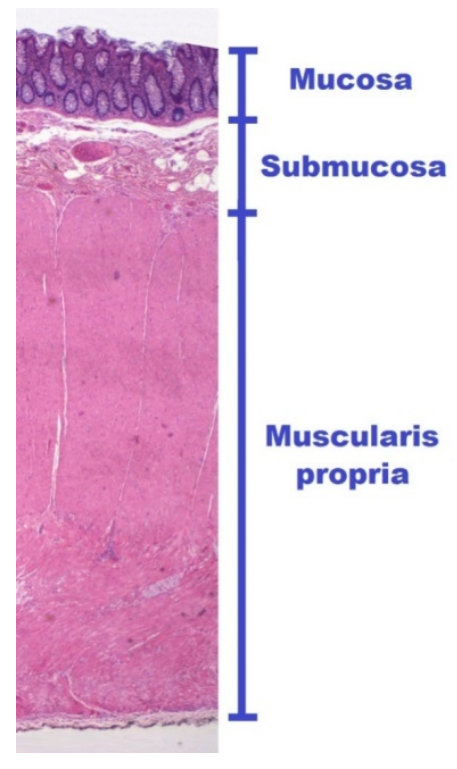

a

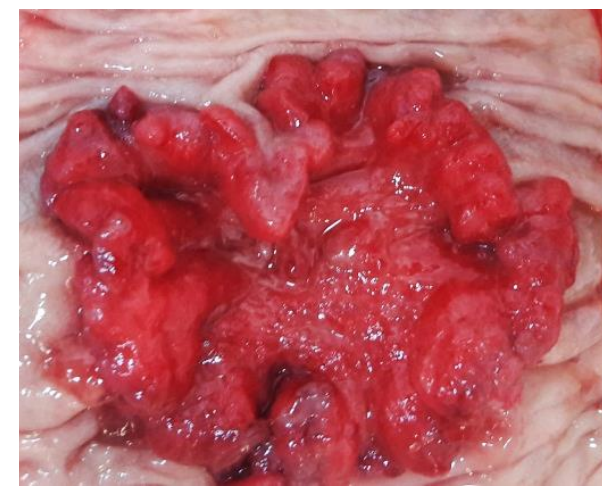

b

Fig. 1 Human colorectal histology section showing the distinct layers that compose its wall (a) and the gross features of an invasive colorectal adenocarcinoma viewed in a surgical specimen (b).

Two types of experimental studies were performed with normal colorectal mucosa, colorectal muscularis propria and pathological tissues.

The first of these studies was the RI measurement using the total internal reflection method and various lasers with different wavelengths between 400 and 850 $\mathrm{nm}$ [29-33]. The samples used in these measurements were prepared with an approximated rectangular shape $\left(\sim 4 \times 3 \mathrm{~cm}^{2}\right)$. These samples were flattened on one side to adhere perfectly to the prism surface. Three samples of each tissue type were used in these measurements to average results.

To estimate the free water content in the various tissues we have performed another study. For colorectal muscle samples under treatment with water-glycerol solutions with different osmolarities we measured the time dependence of collimated transmittance $\left(T_{\mathrm{c}}\right)$ spectra. Using this data and similar time dependencies for normal colorectal mucosa and pathological tissues that were already published [11], we have performed calculations to obtain the free water values in each tissue. The calculations with this data are described in Section 2.3. Similarly to the $T_{\mathrm{c}}$ study published in Ref. 11 , the colorectal muscle samples to use in this study were prepared with circular form $(\phi \cong 1 \mathrm{~cm})$ and $0.5 \mathrm{~mm}$ thickness. To prepare the samples with these dimensions and to flatten the ones for RI measurements, a cryostat from Leica $^{\mathrm{TM}}$, model CM $1850 \mathrm{UV}$ was used. Three samples of each type of tissue were used in each particular $T_{\mathrm{c}}$ study to average results, as indicated in Sub-section 2.3.

\subsection{RI dispersion estimation}

For each type of tissue considered in this research, the total internal reflection method [29-33] was used to measure the RI at different laser wavelengths within the UV-NIR range. In these measurements, we have used lasers with the following wavelengths: 401.4, 532.5, 668.1, 782.1, 820.8 and 850.7 $\mathrm{nm}$ [23].

After obtaining the mean RI values from each type of tissue at these wavelengths, we have used CFTOOL in MATLAB ${ }^{\mathrm{TM}}$ to fit that data with the curves that are commonly used for biological tissues [34-35]. For all tissues we obtained the corresponding dispersion curves between 400 and $1000 \mathrm{~nm}$ and the best data fitting was found with the Cornu equation [34-35]:

$$
n_{\text {tissue }}(\lambda)=A+\frac{B}{\lambda-C} .
$$

In Eq. (3), $\lambda$ represents the wavelength, to be used in nanometers and the Cornu coefficients $\mathrm{A}, \mathrm{B}$ and $\mathrm{C}$ were estimated for each tissue during data fitting.

The calculated dispersion curves were later used to estimate the dispersion curves for tissue scatterers, as described in Sub-section 2.4.

\subsection{Free water content estimation}

To estimate the free water content in colorectal muscle, we have performed several OC treatments with glycerol solutions containing different osmolarities. The same procedure has been previously done for colorectal healthy and pathological mucosa under treatment with glucose solutions (see Ref. [11]). Colorectal muscle samples were treated with glycerol solutions with 
concentrations and osmolarities as represented in Table 1. The osmolarities for the solutions were calculated using Eq. (2) and the molar mass of glycerol $\left(M=92.0776 \times 10^{-3} \frac{\mathrm{Kg}}{\mathrm{mol}}[36]\right)$. In these calculations, the reported [37] densities for glycerol-water solutions at $20^{\circ} \mathrm{C}$ were also used.

Table 1 Glycerol concentrations in water and solution osmolarities.

\begin{tabular}{cc} 
Glycerol concentration (\%) & Osmolarity (osmol/l) \\
\hline 20 & 11.37 \\
25 & 11.51 \\
30 & 11.64 \\
35 & 11.79 \\
40 & 11.94 \\
45 & 12.09 \\
50 & 12.23 \\
55 & 12.38 \\
60 & 12.53
\end{tabular}

Considering these solutions, three studies were performed for each tissue/treatment combination to average final results. During these treatments, the $T_{\mathrm{c}}$ spectra of muscle tissues were acquired between 400 and $1000 \mathrm{~nm}$. Since between 600 and $800 \mathrm{~nm} T_{\mathrm{c}}$ increases linearly for the muscle, we calculated the $T_{\mathrm{c}}$ time dependence curves for individual wavelengths within this range [11].

Considering a particular tissue/treatment study, the averaged $T_{\mathrm{c}}$ time dependence for each particular wavelength was first displaced to have $T_{\mathrm{c}}=0$ at $t=0$ (natural tissue) and then normalized to the highest $T_{\mathrm{c}}$ value observed at the beginning of the saturation regime. This displaced and normalized data was then fitted with Matlab's CFTOOL, with a curve as described by Eq. (2) $[5,11,38]$ :

$$
T_{c}(\lambda, t) \cong\left[1-\exp \left(-\frac{t}{\tau}\right)\right]
$$

Eq. (4) indicates that the time-dependence of the $T_{\mathrm{c}}$ spectra $\left(T_{\mathrm{c}}(\lambda, t)\right)$ describes an exponential increase, which is created by the net effective flow involved in the treatment with a characteristic diffusion time, $\tau$.

During the data fitting, a $\tau$ value was estimated for each wavelength. The mean and standard deviation for this parameter were calculated for each treatment.

The mean $\tau$ values obtained for each OCA concentration were represented as a function of OCA concentration in the treating solution. A smooth spline was created to interpolate between the experimental points and to show the dependence between $\tau$ and OCA concentration in solution. For any particular treatment, the estimated $\tau$ value represents the diffusion time for a combination of two fluxes: water flowing into (for low concentrated glycerol solutions) or out (for high concentrated glycerol solutions) and glycerol flowing only into the tissue. By calculating a spline curve that interpolates the data points, we will see that such curve presents a maximum. Such maximum occurs for a particular glycerol concentration in solution. This particular solution is the one that will have the same water as the free water in the tissue and consequently no water flows in or out the tissue. Consequently, the $T_{\mathrm{c}}$ time dependence that would be measured for a treatment with such solution would be associated only to the glycerol flow into the tissue. Such time dependence has the slowest $\tau$ value that characterizes glycerol diffusion in the tissues and it corresponds to the maximum in the spline curve.

On the other hand, since the unique glycerol flow into the tissue occurs for a treatment with a solution that has the same water content as the free water in the tissue $[11,38]$, we can identify the free water content in the tissue from the graph. As example, if the peak in the graph occurs for a glycerol concentration of $40 \%$, then the water in the treating solution is $60 \%(100 \%-40 \%)$. This way the free water in the tissue will also be $60 \%$. Using this knowledge, we estimated the free water content in each type of tissue.

\subsection{Calculation of the dispersion for tissue scatterers}

The estimated free water contents and dispersion curves from the various tissues were used to calculate the dispersions of tissue scatterers.

To perform these calculations and since our measurements were made at temperatures between 19 and $21^{\circ} \mathrm{C}$, we started by collecting the water dispersion data between 400 and $1000 \mathrm{~nm}$ at $20^{\circ} \mathrm{C}$ from literature [39]. Using this data, we calculated the corresponding dispersion curves for tissue scatterers by subtracting the free water contribution from the experimental dispersion curves of the different tissues. In these calculations we used an adapted version of Gladstone and Dale equation [20]:

$$
\begin{aligned}
& n_{\text {scatterer }}(\lambda)= \\
& =\frac{n_{\text {tissue }}(\lambda)-n_{\text {water }}(\lambda) f_{\text {free-water }}}{1-f_{\text {free-water }}}
\end{aligned}
$$

In Eq. (5), $n_{\text {tissue }}(\lambda)$ is the calculated dispersion for a particular tissue, $n_{\text {water }}(\lambda)$ is the water dispersion retrieved from literature [39] and $f_{\text {free-water }}$ represents the free water content in the tissue. All the results from the sequential calculation steps are presented in Section 3.

\section{Results and discussion}

As indicated in Section 2, our research is based on different measurements and involves some calculation procedures. Therefore, we have structured the presentation of results to show the sequential steps that were performed to obtain the results. The RI measurements and calculations to obtain tissue dispersion curves are presented in Sub-section 3.1. Subsection 3.2 presents the $T_{\mathrm{c}}$ measurements and 
calculations to estimate the free water content in the various tissues and Sub-section 3.3 presents the final calculations to obtain the dispersion curves for tissue scatterers.

\subsection{RI dispersion of colorectal tissues}

According to the description presented in Sub-section 2.2 , the RI measurements made from the various colorectal tissues were made using the total internal reflection method and different lasers.

The samples to use in these measurements have been previously flattened with a cryostat, so they could be placed in perfect contact with the prism surface, without the creation of air bubbles in-between. Considering each laser used in these measurements, we have performed three sets of measurements from three individual samples of each tissue. These measurements were performed using $1^{\circ}$ angular resolution and three reflectance curves at the prism/tissue interface were calculated. Fig. 2 presents these curves for the case of colorectal muscle and for the $532.5 \mathrm{~nm}$ laser:

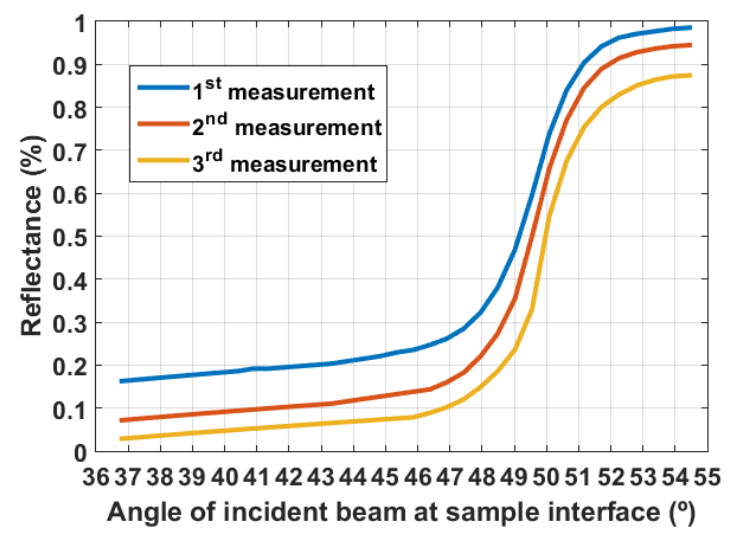

Fig. 2 Reflectance curves at the tissue/prism interface for the measurements with the $532.5 \mathrm{~nm}$ laser.

Calculating the first derivative for each curve in Fig. 2, we obtained the curves presented in Fig. 3, where a significant peak can be seen for each curve:

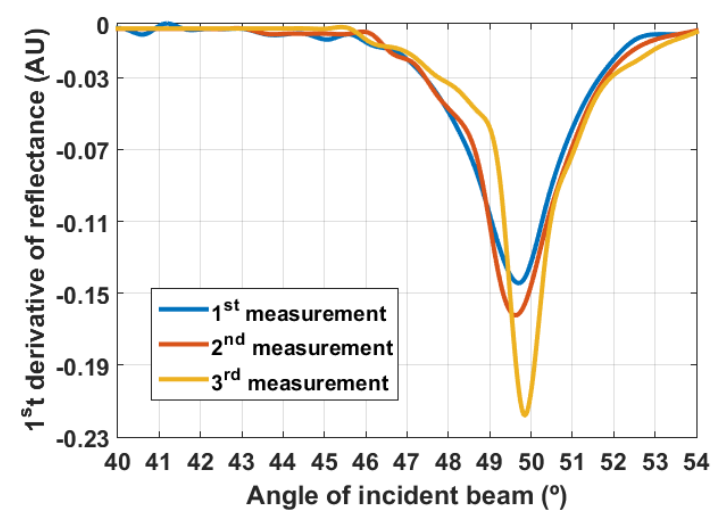

Fig. $31^{\text {st }}$ derivative curves of the reflectance measurements presented in Fig. 2.
From Fig. 3, we have identified the angles where the peaks occur. These angles represent the critical reflection angles at the prism/tissue interface for each set of measurements. To calculate the RI values of the tissue $n_{\text {tissue }}(\lambda)$ for the wavelengths $(\lambda)$ of the lasers used in these measurements, we have used Eq. (6) [23]:

$$
n_{\text {tissue }}(\lambda)=n_{1}(\lambda) \times \sin \left(\theta_{c}\right)
$$

In Eq. (6), $n_{1}(\lambda)$ represents the RI of the prism used in the measurements for the same wavelength of the laser and $\theta_{\mathrm{c}}$ is the critical angle retrieved from the peaks in graphs like the one in Fig. 3.

A mean RI value and correspondent standard deviation (SD) value were calculated for the tissue at each laser wavelength. The mean RI and SD values for all tissues and wavelengths are presented in Table 2.

The data in Table 2 only corresponds to a wavelength range between approximately 400 and 850 $\mathrm{nm}$. Since we wanted to obtain the dispersion curves for the tissues for a wider range, we have considered experimental data from another group [24] at 960 and $1551 \mathrm{~nm}$ to calculate the curves between 400 and 1000 $\mathrm{nm}$. This procedure was made for the normal mucosa and pathological tissue. For the case of colorectal muscle, no data was available, so we have just extended the curve that fits our data.

The equations obtained for the dispersion curves of the three types of tissues for the wavelength range between 400 and $1000 \mathrm{~nm}$ are the following:

$$
\begin{gathered}
n_{\text {mucosa }}(\lambda)=1.315+\frac{16.73}{\lambda-38.84}, \\
n_{\text {muscle }}(\lambda)=1.3346+\frac{19.48}{\lambda+27.17}, \\
n_{\text {pathological }}(\lambda)=1.315+\frac{19.06}{\lambda-49.42} .
\end{gathered}
$$

All these equations are according to Cornu equation (Eq. (3)) [23], and were obtained with an R-square value of 0.999 . The data points in Table 2 and the curves described by Eqs. (7), (8) and (9) are represented in Fig. 4.

The dispersion curves presented in Fig. 4 for normal mucosa and pathological tissue were calculated by neglecting the experimental points at 820.8 and 850.7 $\mathrm{nm}$. Those experimental values are a little higher than the calculated curves, presenting the evidence of lipid content in these tissues $[23,25]$. Since in this wavelength range we have performed measurements only with two lasers, it is not possible to make an accurate characterization of the wavelength dependence for the RI of these tissues due to the presence of lipids. As an alternative, we have represented the curves described by equations 7 and 9 with dashed lines between 784 and $870 \mathrm{~nm}$ [23]. In later calculations to 
Table 2 Experimental RI data of healthy and pathological colorectal tissues at different wavelengths.

\begin{tabular}{ccccccc} 
& \multicolumn{2}{c}{ Normal mucosa } & \multicolumn{2}{c}{ Normal muscle } & \multicolumn{2}{c}{ Pathological tissue } \\
\hline $\boldsymbol{\lambda}(\mathbf{n m})$ & Mean RI & SD & Mean RI & SD & Mean RI & SD \\
\hline 401.4 & 1.3612 & 0.0012 & 1.3801 & 0.0012 & 1.3693 & 0.0017 \\
532.5 & 1.3489 & 0.0013 & 1.3693 & 0.0024 & 1.3545 & 0.0011 \\
668.1 & 1.3415 & 0.0010 & 1.3626 & 0.0014 & 1.3460 & 0.0012 \\
782.1 & 1.3376 & 0.0012 & 1.3590 & 0.0018 & 1.3411 & 0.0008 \\
820.8 & 1.3393 & 0.0012 & 1.3575 & 0.0014 & 1.3424 & 0.0009 \\
850.7 & 1.3370 & 0.0012 & 1.3566 & 0.0014 & 1.3408 & 0.0011
\end{tabular}

estimate the dispersion curves for tissue scatterers, we will use the tissue dispersion curves as described by equations 7,8 and 9 for the entire spectral range between 400 and $1000 \mathrm{~nm}$.

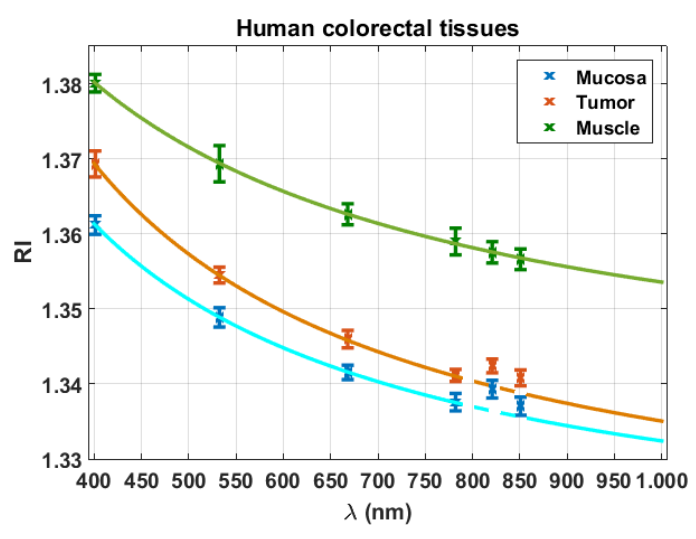

Fig. 4 Experimental RI data and calculated dispersion curves for colorectal tissues.

\subsection{Determination of the free water content in colorectal tissues}

To calculate the dispersion curves for scatterers in colorectal tissues, the free water content is necessary. The evaluation of the free water content was made from measurements of tissue's $T_{\mathrm{c}}$ spectra during OC treatments.

As described in Sub-section 2.3, we have prepared several colorectal tissue samples with $0.5 \mathrm{~mm}$ thickness to use in these studies. The normal mucosa and pathological tissues were previously treated with waterglucose solutions with different osmolarities [11] and the muscle samples were treated in the present research with water-glycerol solutions, also with different osmolarities.

$T_{\mathrm{c}}$ time dependencies for individual wavelengths between 600 and $800 \mathrm{~nm}$ were obtained for all tissue/treatment studied. Fig. 5 presents those time dependencies for the treatments of muscle with $20 \%$, $40 \%$ and $60 \%$-glycerol:

For each particular treatment, the $T_{\mathrm{c}}$ time dependencies before the beginning of the saturation regime were vertically displaced to have $T_{\mathrm{c}}=0$ at $t=0$ and then normalized to the highest value [11]. This rearranged data was fitted with a curve described by Eq. (4). When performing these fittings, the $\tau$ values were estimated for each wavelength within a particular treatment. Graphs representing the mean $\tau$ vs OCA concentration were created for each tissue. The graphs for normal mucosa and pathological tissues are presented in Ref. [11].

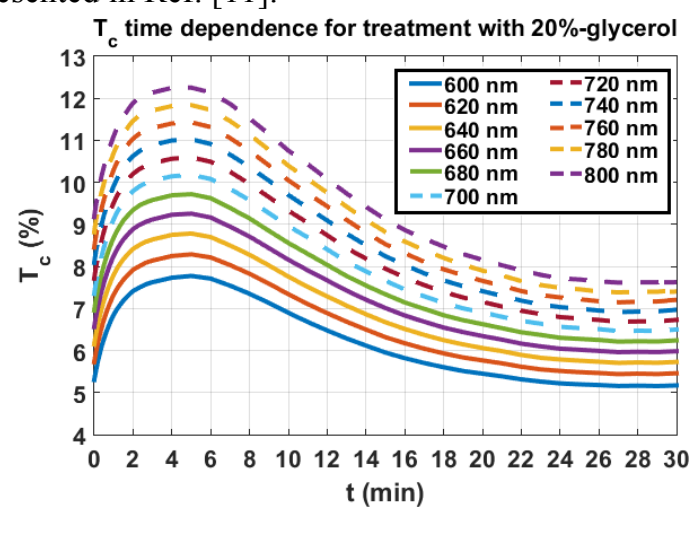

a

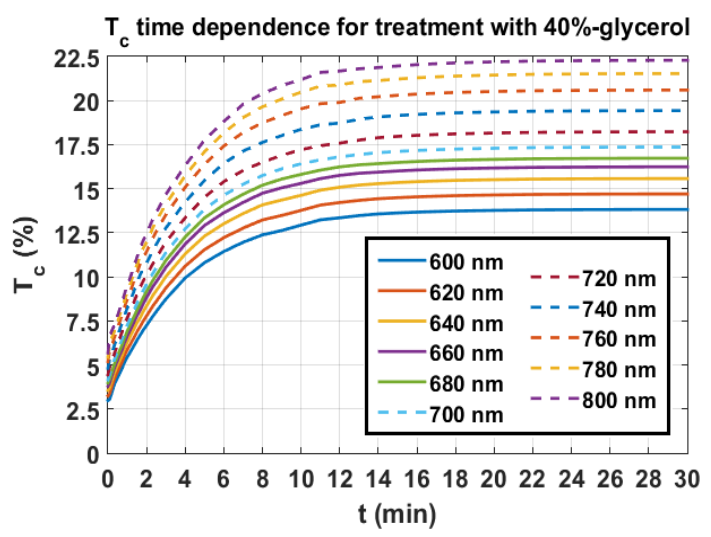

b

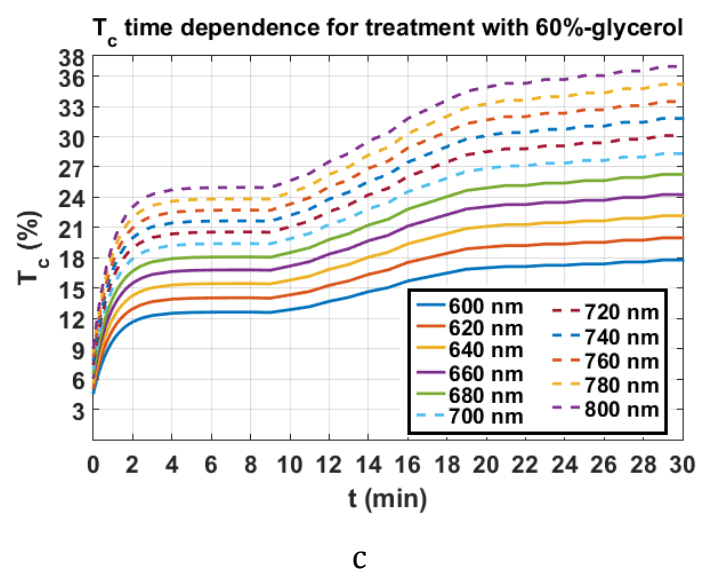

Fig. $5 T_{\mathrm{c}}$ Time dependencies for the treatments of colorectal muscle with: 20\%-glycerol (a), 40\%-glycerol (b) and 60\%-glycerol (c). 
Fig. 6 represents the case of colorectal muscle:

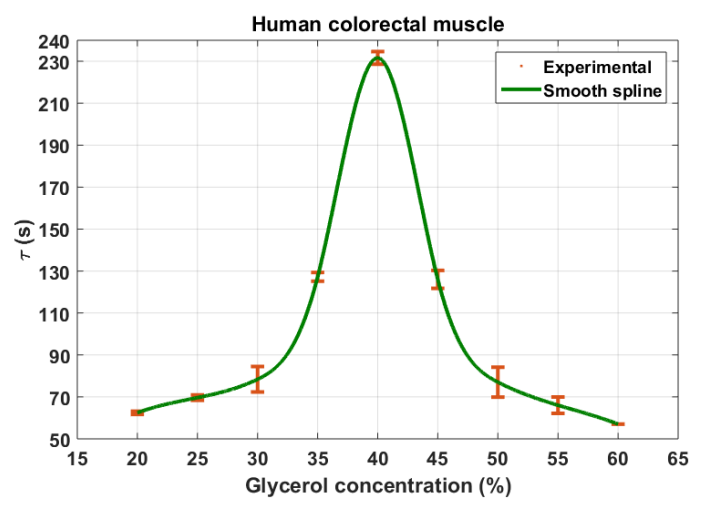

Fig. 6 Mean diffusion time as a function of glycerol concentration in the treating solution.

The peak in the graph of Fig. 6 corresponds to a unique diffusion of glycerol molecules into the muscle tissue. In this case the water in the solution is in perfect balance with the free water inside the tissue, meaning that by identifying the glycerol concentration in the graph of Fig. 6 as $40 \%$, the free water content in the tissue can be calculated as: $100 \%-40 \%=60 \%$. Similar estimations of the free water content in normal mucosa and pathological tissue were also made [11]. The estimated values were: 0.594 (normal mucosa), 0.644 (pathological tissue) and 0.600 (muscle).

These values can now be used with the dispersion curves presented in Sub-section 3.1 in calculations to obtain the dispersion curves of tissue scatterers.

\subsection{Calculation of the dispersion curves for scatterers in colorectal tissues}

Using the dispersion curves of the colorectal tissues that are described by Eqs. (7), (8) and (9) and the free water content values in Eq. (5), we have calculated the dispersion curves for the tissue scatterers. Considering each tissue, these calculations are described by Eqs. (10), (11) and (12):

$$
\begin{aligned}
& n_{\text {scat-mucosa }}(\lambda)= \\
& =\frac{n_{\text {mucosa }}(\lambda)-n_{\text {water }}(\lambda) \times 0.594}{1-0.594}, \\
& n_{\text {scat-muscle }}(\lambda)= \\
& =\frac{n_{\text {muscle }}(\lambda)-n_{\text {water }}(\lambda) \times 0.600}{1-0.600}, \\
& n_{\text {scat-pathological }}(\lambda)= \\
& =\frac{n_{\text {pathological }}(\lambda)-n_{\text {water }}(\lambda) \times 0.644}{1-0.644} .
\end{aligned}
$$

The dispersion curves for tissue scatterers obtained in these calculations are presented in Fig. 7:

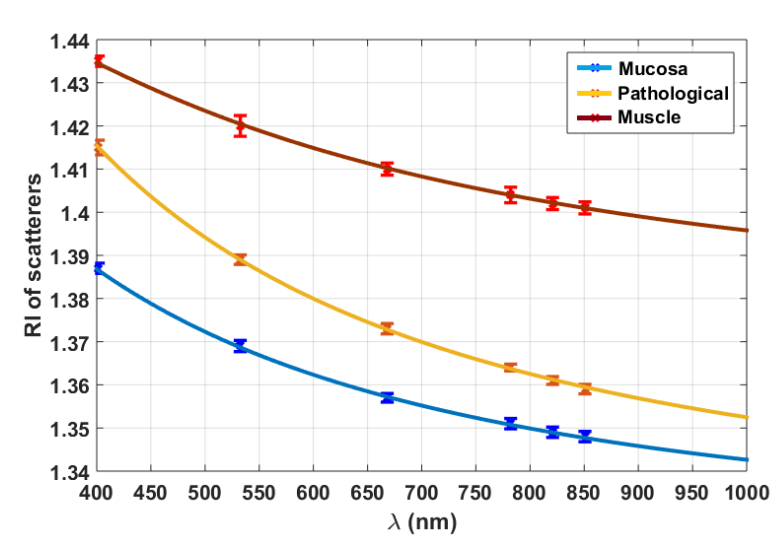

Fig. 7 Wavelength dependence of scatterers RI in colorectal tissues.

The dispersion curves presented in Fig. 7 show differences between the three tissues studied. First, comparing between normal mucosa and muscle, we see that the muscle dispersion shows higher values than mucosa. Such difference might be related to the different nature of the dry matter content in the two tissues, or also to the fact that mucosa might have higher bound water content than muscle. The dispersion for pathological colorectal tissue is also different from the other two, meaning that colorectal pathology is optically distinct from healthy tissues and ex vivo examination of the refractive dispersion may serve for diagnostic purposes.

These curves are also important for OC treatment planning in colorectal tissues. Since in the case of shorttime OC treatments, only free water will be involved in the fluid exchange, the curves in Fig. 7 will remain unchanged during treatments. By performing some calculations with the data in curves of Fig. 7, it is possible to quantify and characterize the RI matching mechanism in OC treatments. Such calculations are described in literature [5], and in one case that we have studied recently for skeletal muscle under treatment with glucose and ethylene glycol [19].

\section{Conclusion}

The refractive dispersion curves for scatterers in colorectal tissues were obtained using a simple method based on direct RI and $T_{\mathrm{c}}$ time dependence measurements during OC treatments.

The $T_{\mathrm{c}}$ measurements performed during treatments allowed to calculate the free water contents in different colorectal tissues, and although values are in the same order of magnitude, the method is sensitive enough to discriminate between different tissues. We also observed that the pathological tissue presents considerably higher water content than normal tissues.

Combining these values with the experimental tissue dispersions, it was possible to calculate the dispersion curves of scatterers in colorectal tissues. These dispersion curves remain unchanged during OC treatments and may be used to evaluate the RI change in 
tissue fluids or to characterize the RI matching mechanism and OC efficiency.

In the near future we plan to perform those calculations and perform similar research for different tissues.

\section{Disclosures}

The authors declare that there are no conflicts of interest related to this article.

\section{Acknowledgements}

This research was supported by the Portuguese research grant FCT-UID/EQU/04730/2013.

VVT was supported by the RF grants: Governmental 14.Z50.31.0004; RFBR 17-02-00358, and MES17.1223.2017/AP. 